\title{
Myelination of the Pig's Brain: A Correlated MRI and Histological Study
}

\author{
Marong Fang a Jicheng $\mathrm{Li}^{\mathrm{a}}$ Xiangyang Gong ${ }^{\mathrm{b}}$ Gregory Antonio ${ }^{c}$ Frances Lee ${ }^{\mathrm{d}}$ \\ W.H. Kwong ${ }^{d}$ S.M. Wai ${ }^{d}$ D.T. Yew ${ }^{d}$ \\ Departments of a Anatomy and ${ }^{b}$ Radiology, Zhejiang University, Hangzhou, PR China; ${ }^{c}$ Department of \\ Diagnostic Radiology and Organ Imaging, Chinese University of Hong Kong, Prince of Wales Hospital, Hong Kong, \\ and ${ }^{\mathrm{d} D e p a r t m e n t}$ of Anatomy, Chinese University of Hong Kong, Shatin, N.T., Hong Kong
}

\section{Key Words}

$\mathrm{T}_{1}$-weighted image $\cdot$ Magnetic resonance imaging • High signal-intensity spots · Myelination · Central nervous system

\begin{abstract}
Minipigs, 2, 4, 6 months old, were used to evaluate the relationship between myelination in the fiber tracts of the central nervous system (CNS) of this animal during development. Histological results showed an increased density of the myelinated fibers as well as branching of these fibers in the areas studied, including the cortical white matter, olfactory tract, the corticospinal tract, the fasciculus cuneatus and the spinal $V$ nucleus from 2 to 6 months old. By 6 months, the pig was sexually matured. Concomitantly, there was an increase in high signalintensity regions (sites) in the magnetic resonance $\mathrm{T}_{1}$ weighted images as myelination progressed. There is a good correlation between the histologically observed progress of myelination and the $\mathrm{T}_{1}$-weighted images in the development of the CNS of the pig.
\end{abstract}

Copyright $\odot 2005$ S. Karger AG, Basel

\section{Introduction}

Axonogenesis, synaptogenesis and myelination are important events in the development of the central nervous system (CNS). Myelination of the nerve fibers is executed by oligodendrocytes [1] and animals with abnormal myelin development or demyelination have severely impaired pathways [2], leading to behavioral defects such as changes in locomotive function or impaired coordination [3]. Unfortunately, in the postnatal development of the CNS of experimental animals, the temporal sequence of myelination has been largely neglected. Due to its similarity with humans the pig is an ideal animal for experimental research $[4,5]$. However, its development of the CNS, especially the myelination of fibers, remains undocumented. We report here some of the changes in myelination of the CNS of the pig during infant and young adult development.

On the other hand, it is generally accepted that magnetic resonance (MR) imaging can be used to visualize myelination of the brain [6-12]. Myelination can be identified based on $T_{1}$ or $T_{2}$-weighted MR imaging reflecting the difference in tissue water. $\mathrm{T}_{1}$-weighted imaging gives a high-intensity signal whereas $T_{2}$ gives an inverted signal (hypointensity) [13]. On this basis, $T_{1}$ and $T_{2}$-weighted MR imaging as well as diffusion-weighted MR imaging have been employed in recent years to study brain development in both human fetuses and young children [1423]. Utilizing this special feature of MR and accepting the changes in the intensities of the tracts reflected in myelination, we therefore histologically compared the myelination of the CNS of the pig using $\mathrm{T}_{1}$-weighted $\mathrm{MR}$ imaging in our observations. In the maturing of the brain white matter, a significant decrease in water content leads to a decrease in longitudinal relaxation times $\left(\mathrm{T}_{1}\right)$ and transverse relaxation times $\left(\mathrm{T}_{2}\right)$. The onset of myelination

\section{KARGER \\ Fax +41613061234 E-Mail karger@karger.ch} www. karger.com (c) 2005 S. Karger AG, Base

$1424-862 X / 05 / 0143-0102 \$ 22.00 / 0$

Accessible online at: www. karger.com/nsg
D.T. Yew

Department of Anatomy, Chinese University of Hong Kong

Shatin, New Territories

Hong Kong SAR (China)

Tel. +852 2609 6899, Fax +852 2603 5031, E-Mail david-yew@cuhk.edu.hk 
leads to the increase in lipid content which can further induce differences in grey-white matter contrast in $T_{1}$ and $\mathrm{T}_{2}$-weighted images during the course of myelination [15]. However, it still remains controversial whether $T_{1^{-}}$ or $\mathrm{T}_{2}$-weighted MR imaging is better for the detection of myelination changes, and there have been pros and cons for both $T_{1}$ - or $T_{2}$-weighted $M R$ in this area of research [16]. An excellent study by Baratti et al. [17], comparing MR imaging in brain maturation in kittens with $T_{1}$ or $T_{2-}$ weighted imaging and trace of diffusion-tensor imaging, reported that the 'long $\mathrm{T}_{2}$ component and trace $\mathrm{D}$ could discourage the use of diffusion MR sequences' in brain development studies. In this work, we regard that the conventional MR image sequence is more easily done and the results are more comparative to former studies.

\section{Materials and Methods}

\section{Animal Care}

Fifteen female Bama minipigs, aged from 2 to 6 months (2 months old, $n=4 ; 4$ months old, $n=5 ; 6$ months old, $n=6$ ), weighing from 2.5 to $17 \mathrm{~kg}$, were obtained from the Laboratory Animal Centre for minipigs in Guangxi Medical University, China. The pigs were housed in large cages, 1 to a cage, and maintained at $22^{\circ} \mathrm{C}$ on a $12 /$ 12-hour light-dark cycle. All animals were cared for in accordance with the guidelines issued by the ethical committees of the Chinese University of Hong Kong and Zhejiang University.

\section{Histology}

All pigs were killed with an overdose of sodium pentobarbital $(1 \mathrm{~g} / \mathrm{kg})$ after MR imaging recording. In order to ascertain whether there was any change in the myelination of the CNS with age, the olfactory tract, the white matter of the cortex (prefrontal region) and the brainstem areas were dissected out from the brains of pigs at 2, 4, and 6 months and cut into small pieces. These specimens represented different selected areas of the CNS. They were then initially fixed in $4 \%$ paraformaldehyde for $24 \mathrm{~h}$ and were then transferred to $1 \% \mathrm{OsO}_{4}$ for $3 \mathrm{~h}$. Afterwards, the blocks were dehydrated in graded alcohol, cleared in xylene and embedded in paraffin. Coronal and horizontal sections were cut at $6 \mu \mathrm{m}$ and the slides dewaxed in xylene and finally embedded in permount. The areas of the cortical prefrontal white matter, the olfactory tract, the medial lemniscus in the medulla, the corticospinal tracts in the pons, the fasciculus gracilis in the medulla and the spinal trigeminal nuclei (spinal V in the medulla) were selected for observation and evaluation. The logic was to select a representative section from the cortical white matter, visceral sensory, somatic sensory and somatic motor tracts as well as a representative section of the cranial nerve nuclei.

Morphometrically, animals were used at 2, 4, and 6 months of age. The number of myelinated fibers were counted at $\times 400$ magnification in 30 randomly selected fields of 500 cells in each of the above regions from the animals of each age group, except for the spinal trigeminal nucleus for which the number of myelinated fibers invading the nucleus were counted in 30 randomly selected fields $\left(700 \mu \mathrm{m}^{2}\right)$ at $\times 400$. The myelinated fibers were defined as fibers with the myelin sheath stained by osmium tetroxide which is an accepted classical protocol for myelin. The diameters of the fibers were not taken into account. The mean and standard error were computed. Statistical analysis was performed by the one-way ANOVA. The statistical significance level was set at $p \leq 0.05$ in all cases. The numbers of myelinated fibers in the cortical white matter were not counted as they were too numerous.

For $\mathrm{T}_{1}$ MR imaging, a special custom-built holder for the pig's body and the head was developed with Plexiglas materials. A foam headrest was made to restrain movement of the head in all directions. Our design provided a rigid detailed mold for the head and body of the pig and minimized motion during the MR imaging studies, but enabled accurate repositioning of the head.

Unanesthetized pigs were trained to adapt to restraint in the animal holder and for the MR image scanning. After a $0.5 \mathrm{~h}$ of adaptation, imaging was performed on a 1.5-T unit (Magnetom Sonata; Siemens, Erlangen, Germany). For the anatomical MR images, slice thickness was $2 \mathrm{~mm}$ and the gap in between $2 \mathrm{~mm}$. There were $150+$ slices in each 6-month specimen, $90+$ slices in each 4-month specimen and $70+$ slices in each 2-month specimen. Slices parallel to the intercommissural line covering the whole brain were imaged with SE sequence $\mathrm{T}_{1}$-weighted imaging with the following parameters: repetition time (TR) $500 \mathrm{~ms}$, and echo time (TE) $14 \mathrm{~ms}$. The field of view (FOV) was $180 \times 180 \mathrm{~mm}$, matrix size $256 \times 256 \mathrm{~mm}, 2$ acquisitions.

In order to quantify the hyperintensity of the signals in the MR image at the different ages, 10 random fields were chosen from the cortical corona radiata, the internal capsule and the brainstem sensory tracts of each animal of each age group and the grey value computed by eFilm software (version 1.5.3, eFilm Medical, Toronto, Ont., Canada). The grey values of white fibers were compared with the grey values within the muscles of the same MR images to avoid any artifact and were expressed finally as a ratio of grey values of white fibers versus grey values of muscles. The final values were compiled and expressed as \pm SE. Significance was set at $p \leq 0.05$.

\section{Results}

Histology of the cortical white matter revealed a grossly visible increase in myelinated areas from 2 to 6 months (fig. 1). By 6 months, histological observations of random areas revealed that all white fibers in the areas were myelinated in the cortical white matter. A comparison of the olfactory nerves indicated the increase in myelinated fibers from 2 to 6 months (fig. 2a, b) and morphometry confirmed an increase in myelinated fibers per unit area $\left(700 \mu \mathrm{m}^{2}\right)$ as the animal aged (fig. $\left.2 \mathrm{c}\right)$. Similar observations were recorded in the corticospinal tract in the pons histologically (fig. 3a, b) and morphometrically (fig. 3c) and in the fasciculus gracilis in the medulla histologically (fig. 4a, b) and morphometrically (fig. 4c).

Inspection of the spinal $\mathrm{V}$ nucleus in the medulla revealed an increase in myelinated fibers invading the nucleus with increasing age (fig. 5a-c) and many of the myelinated fibers in the spinal $\mathrm{V}$ nucleus of the 4- to 6- 
Fig. 1. Histological sections of $\mathrm{OsO}_{4}$-fixed myelinated fibers in cortical white matter at 2 months (a), 4 months (b) and 6 months (c). Note the increase in myelinated fibers, and by 6 months, all observable fibers appeared to be myelinated. $\times 400$.
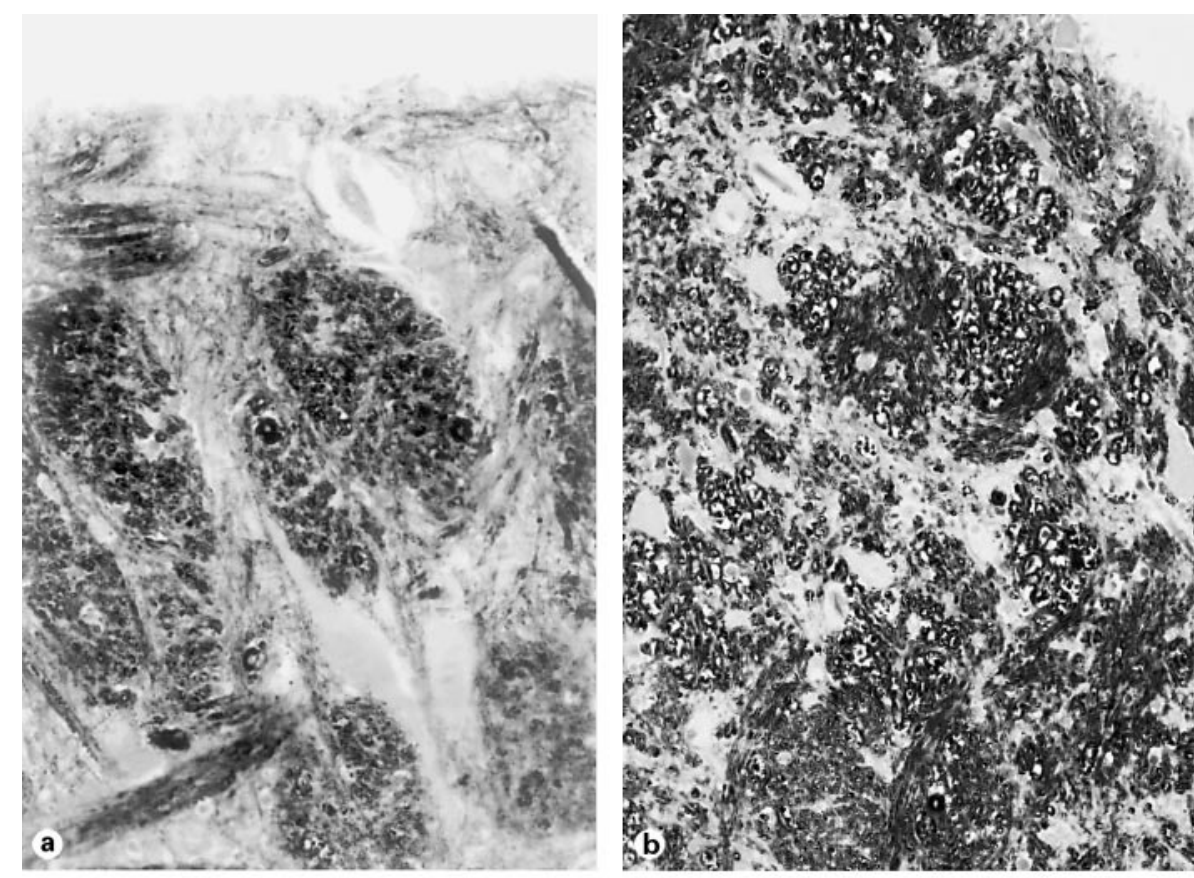

month-old brain actually showed branching (fig. 5b, c). The morphometric comparison between age groups is shown in figure $5 \mathrm{~d}$.

$\mathrm{T}_{1}$-weighted MR images displayed some hyperintense signals in the white fibers all over the prosencephalon and brainstem (fig. 6) at 2 months of age. By 4 months of age, many of the hyperintense signals increased in number (fig. 7, 8) and were most numerous in number in the prosencephalon (telencephalon and diencephalon) and brainstem by 6 months of age (fig. 9). The quantitation of the signal intensity in the selected areas is depicted in table 1. For quantitation in the cortical corona radiata and the internal capsule, significant differences were obtained in the grey matter value between different ages $(p<0.05)$. The medulla did not show a statistically significant difference, probably due to the fine alignment diversity of different pathways. However, there was still an increase in grey value at 6 months old compared with 2 months old.

\section{Discussion}

Our microscopic studies reveal that with advancing age, an increase in density per unit area of myelinated fibers was found in the areas studied during this period. This appeared to correlate with the $\mathrm{T}_{1}$-weighted $\mathrm{MR}$ images in that the increase in hyperintense sites did reflect

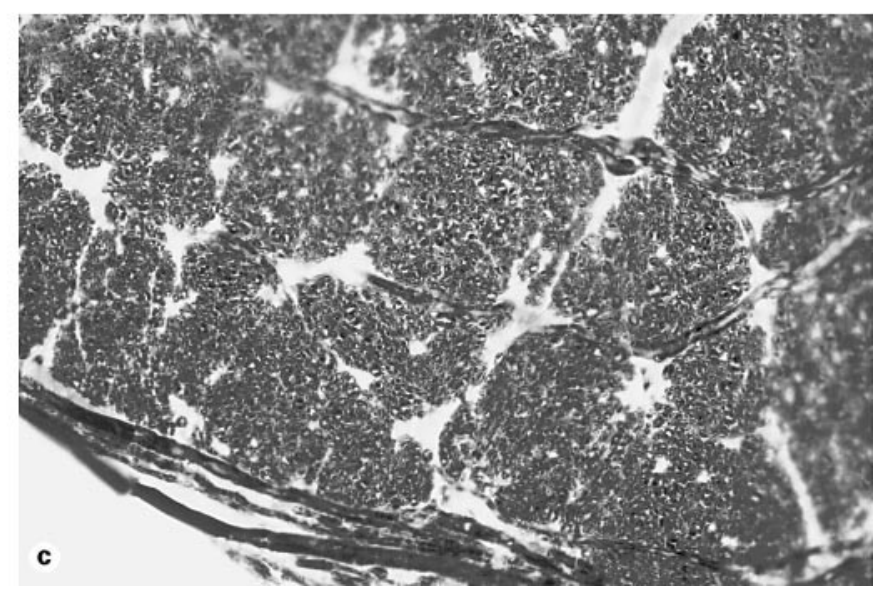

the process of myelination, just as indicated by previous authors [6-13]. Myelination was scanty and at its early stage by 2 months of age, and MR imaging demonstrated individual spotty hyperintense signals in diverse areas of the CNS, e.g. cortical white matter, deep telencephalon (basal ganglia and internal capsule) as well as the brainstem. By 4 months, these areas began to increase in the extent of myelination and demonstrated additional numbers of hyperintense signals, and by 6 months, major CNS myelination was about to be completed and most white fibers were fully myelinated, these areas exhibiting very high numbers in the quantity of hyperintense signals. Since the minipig has a lifespan of 15 years, 6 months should be equivalent to 3 years of age in human, if the 

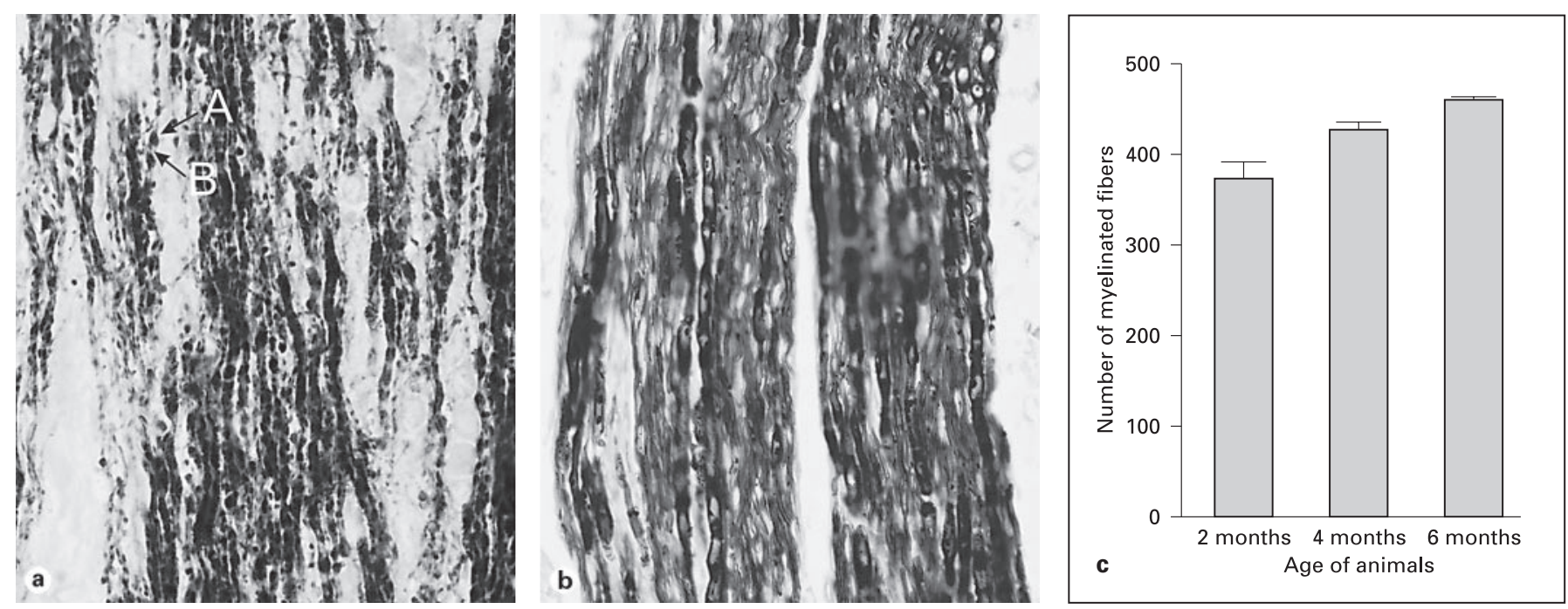

Fig. 2. a Histological section of the olfactory tract of the 2-month-old pig after osmium tetroxide staining which showed some myelinated fibers. The spaces between the myelinated fibers (black) were filled with non-myelinated (or not completely myelinated) fibers. There were also segments of myelination (A) and non-myelination (B). Horizontal section. $\times 400$. b Histological section of the olfactory tract of a 4-month-old pig after osmium tetroxide staining which showed more myelinated fibers. Horizontal section. $\times 400$. $\mathbf{c}$ Histogram showing the number of myelinated fibers per 500 total fibers in the olfactory tract of pigs aged 2, 4 and 6 months. The bars denote the standard error.
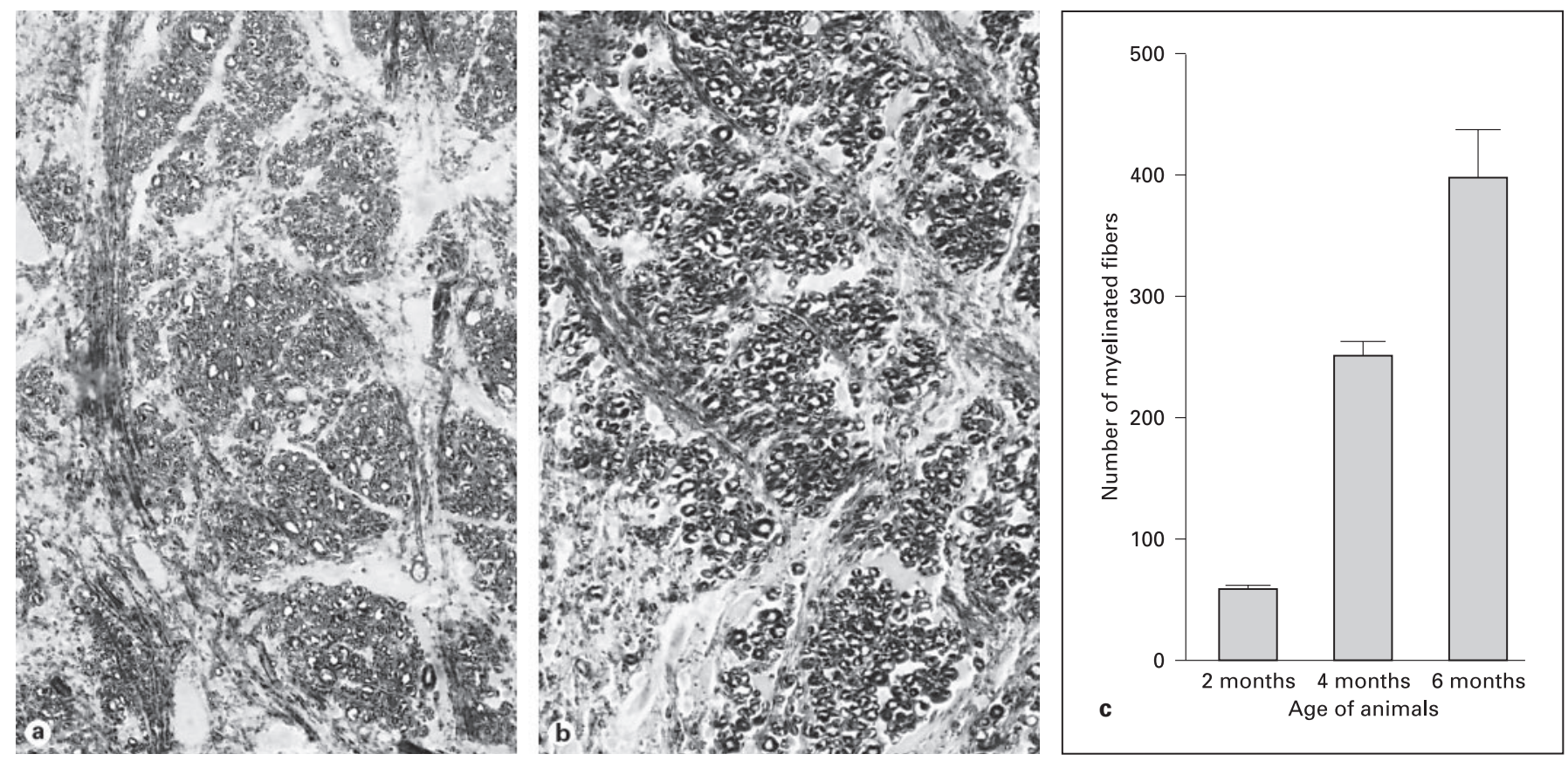

Fig. 3. Histological sections of the corticospinal tracts in the pons of pigs aged 2 (a) and 4 months (b). Myelinated fibers were stained with osmium tetroxide. Horizontal section. $\times 200$. c Histogram showing the number of myelinated fibers per 500 total fibers of the corticospinal tract in the pons of pigs aged 2, 4 and 6 months. The bars denote the standard error. 

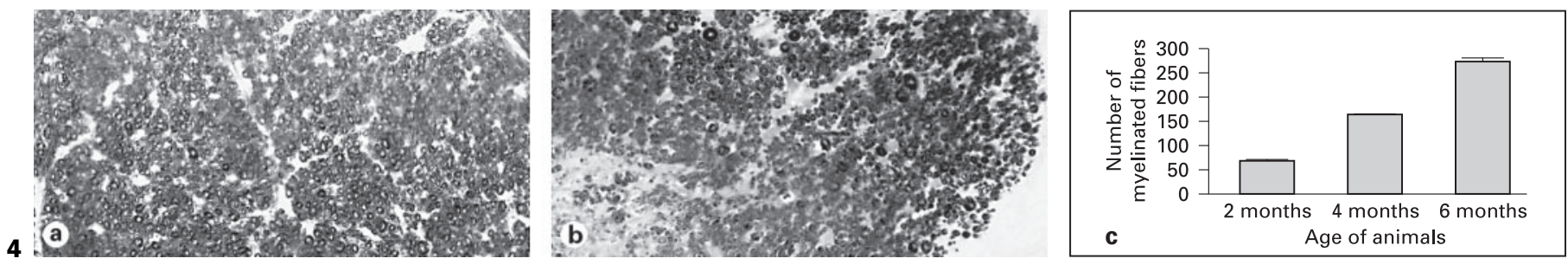
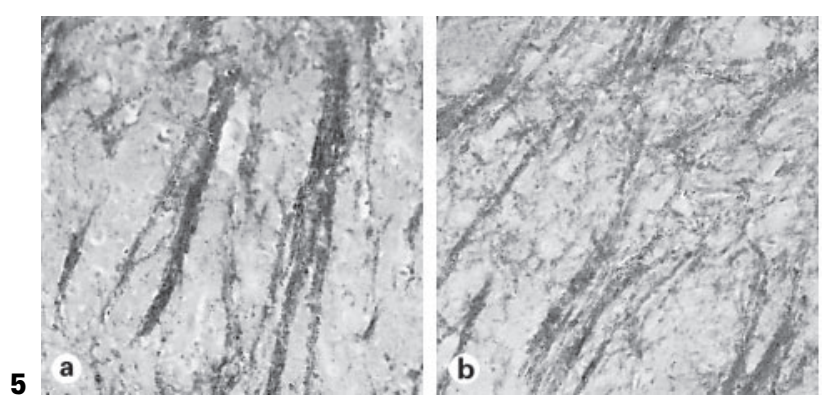

Fig. 4. Histological sections of the fasciculus gracilis in the medulla of pigs aged 2 (a) and 6 months (b). Myelinated fibers were stained with osmium tetroxide. Horizontal section. $\times 400$. c Histogram showing the number of myelinated fibers per 500 total fibers of the fasciculus gracilis in the medulla of pigs aged 2, 4 and 6 months. The bars denote the standard error.
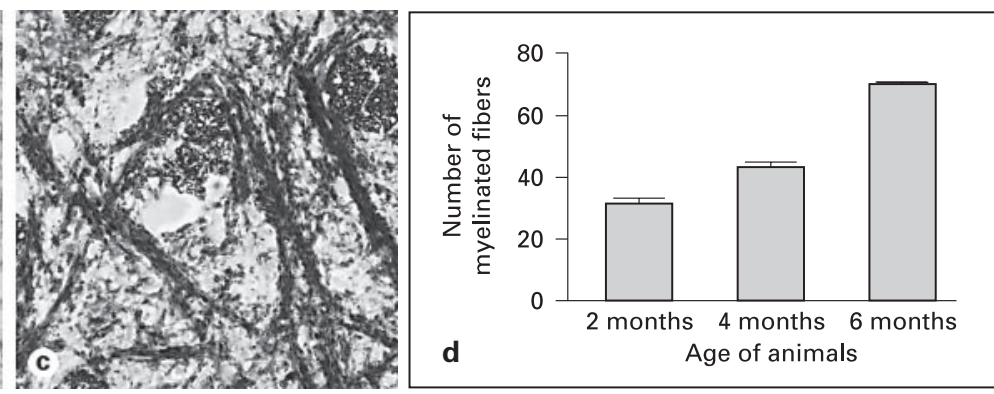

Fig. 5. Histological sections of myelinated fibers stained by osmium tetroxide in the spinal $\mathrm{V}$ nuclei of pigs aged 2 (a), 4 (b) and 6 months (c) old. Note the increase in density and branches in figure $\mathbf{b}$ and $\mathbf{c}$. d Histogram showing the number of myelinated fibers per unit area $(\times 400)$ of the spinal V nuclei of pigs aged 2, 4 and 6 months old. The bars denote the standard error.

Table 1. Grey values of nerve fibers versus grey values of muscles

\begin{tabular}{lllll}
\hline & 2 months & 4 months & 6 months & Statistical significance \\
\hline Cortical corona radiata & $1.305 \pm 0.075$ & $1.570 \pm 0.059$ & $1.884 \pm 0.070$ & Significant difference between 2 and 4, and 4 and 6 months \\
Internal capsule & $1.453 \pm 0.055$ & $1.510 \pm 0.067$ & $1.970 \pm 0.092$ & Significant difference between 4 and 6 months \\
Medulla & $1.461 \pm 0.046$ & $1.420 \pm 0.077$ & $1.534 \pm 0.073$ & - \\
\hline
\end{tabular}

curve is linear. Myelination has been studied in the kitten and was shown to be completed by 3 months of age [17]. Considering that a cat can live to 15 years of age, 3 months is equivalent to 1-2 years old in the human. In the human fetus, between 12 and 34 gestation weeks, myelination begins to appear in different areas in the brainstem, diencephalon and cerebellum but is not significant in the hemispheres $[13,20]$. Myelination continues in the young child [18] well into the second decade [13, 19]. Recent studies suggested that changes in signal intensity from MR imaging might not only refer to myelination but also to the development of nerve cells (e.g. increase in cells) related to these tracts [24]. In our work, however, morphological observation on the neurons and quantitation by PCNA immunocytochemistry (to show dividing cells) on the specimens of different ages did not identify any significant differences in the percentage of dividing new cells between ages in preliminary studies. The great majority of white fibers in the telencephalon and brainstem in the human in fact were myelinated by the first year [24] as shown by $T_{1}$-weighted images. Myelinated fibers were seen in the 2-month-old pig and progressed to 6 months when the pig is sexually mature. The olfactory tract showed a small increase in myelinated fibers between 2 and 6 months of age in the pig, whereas the motor fibers (corticospinal) and sensory fibers (fasciculus gracilis) showed a much greater increase in the number of myelinated fibers. It appears therefore that the peripheral fibers were actually myelinated earlier than the central fibers and that both sensory and motor pathways myelin- 

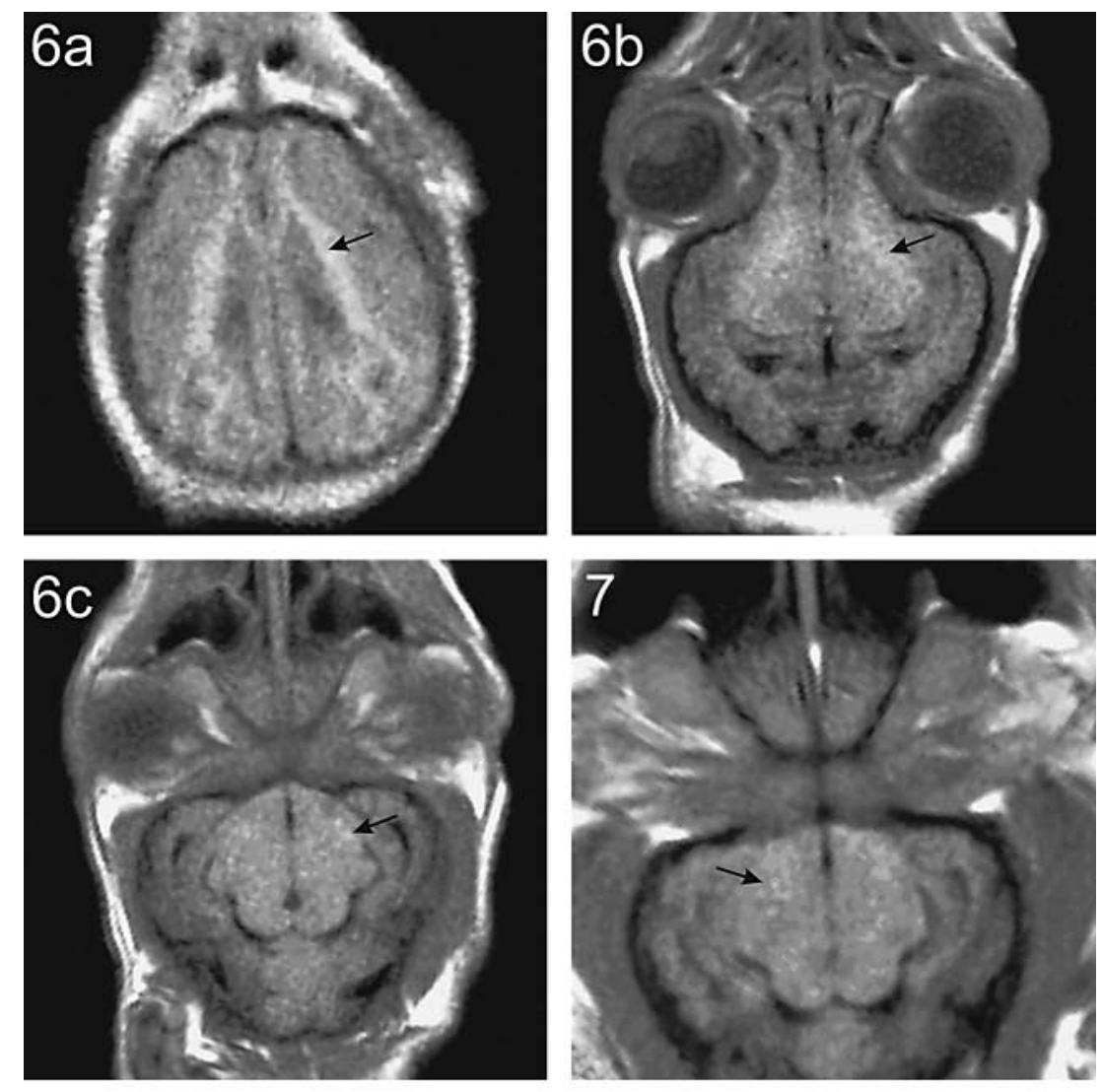

Fig. 6. a, b $T_{1} M R$ image of a 2-month-old pig showing some hyperintense signals (arrows) in the white matter of the cortex and other parts of the prosencephalon. c $\mathrm{T}_{1} \mathrm{MR}$ image of a 2-month-old pig showing some hyperintense signals in the brainstem (arrow).

Fig. 7. $T_{1} M R$ image of a 4-month-old pig showing more hyperintense signals in the brainstem (arrow).

Fig. 8. $T_{1}$ MR image of a 4-month-old pig showing more hyperintense signals in the telencephalon (arrow).

Fig. 9. a $T_{1}$ MR image of 6-month-old pig showing the highest number of hyperintense signals in the cortical white matter (arrows). b, c $\mathrm{T}_{1} \mathrm{MR}$ image of a 6-month-old pig showing the highest number of hyperintense signals (arrows) in the deep part of the prosencephalon (b) and brainstem (c).
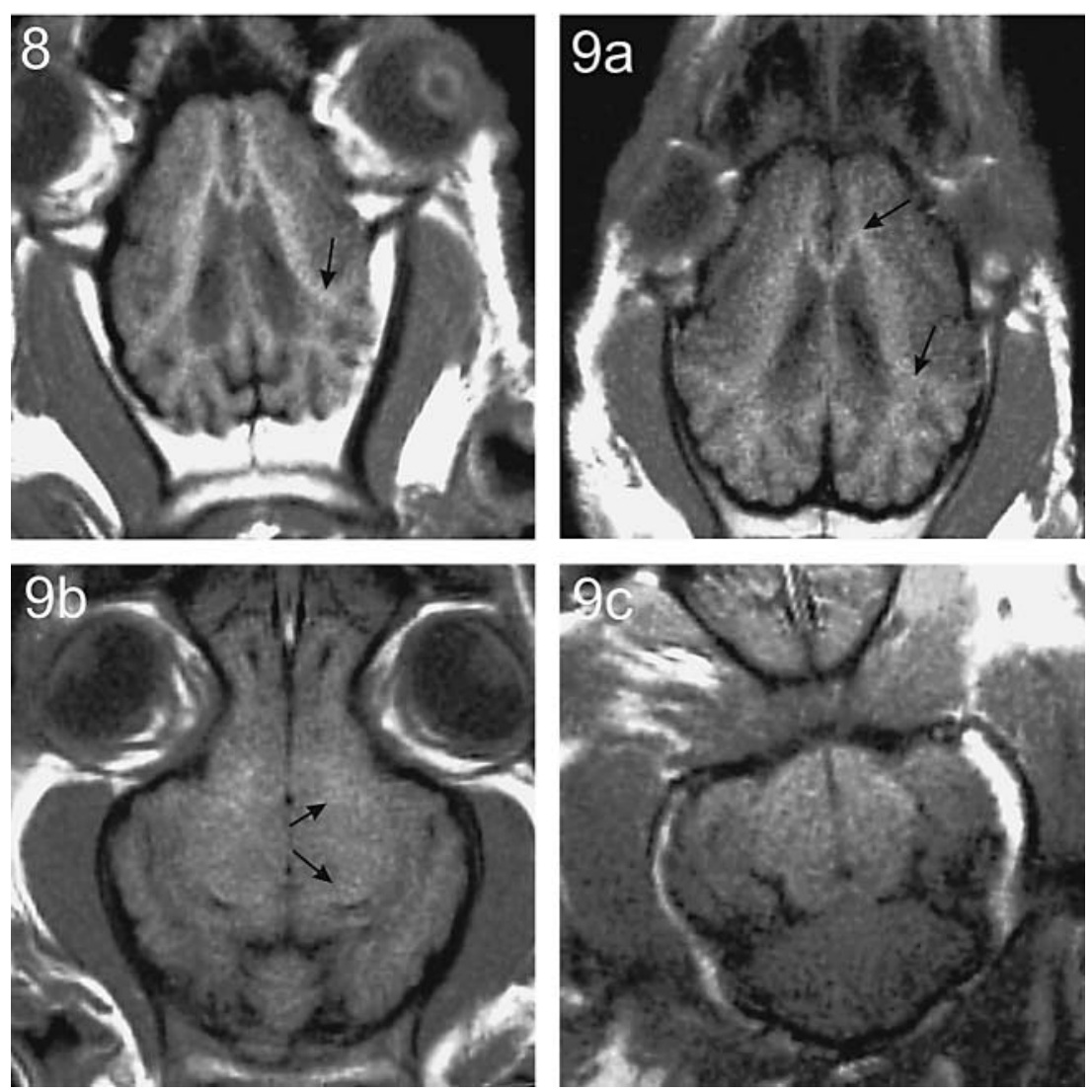
ated at the same rate in the pig. This differs from humans in that the proximal pathways myelinated earlier than the distal and sensory pathways and before the motor pathway, as revealed by autopsy [25]. This is not surprising since the pig has a much shorter lifespan but much earlier sexual maturation, lives in a different environment and faces different challenges from that of the human. Extrapolation of the timing of myelination from the pig to human is therefore difficult.

This is the first experimental study to correlate the hyperintense signals of fiber tracts from $T_{1}$-weighted $M R$ imaging with the histological evidence of myelination of the white fiber tracts in animals. Since pigs are used in many experimental studies $[4,5]$ due to their similarity to the human, knowledge of its myelination will undoubtedly form a background for future CNS studies in this species.

\section{Conclusion}

In this myelination study of the pig's brain, we found that myelination was scanty in the 2-month-old pig but increased in amount up to 6 months of age, a time when the pig is sexually matured. On the whole, our histology results correlated generally well with the $T_{1}$-weighted $M R$ images in that myelination relates to the appearance of hyperintense signals in $\mathrm{T}_{1}$-weighted $\mathrm{MR}$ images. The correlative histology study supplemented with $T_{1}$-weighted MR imaging also shows that not only are $T_{1}$-weighted $M R$ images able to detect myelination as documented, but any changes in myelination in the different sites can be highlighted by MR imaging. The histological plus MR imaging approaches were therefore useful in studying myelination in animals and also helped to fine tune the results of each other.

\section{References}

1 Tsai HH, Miller RH: Glial cell migration directed by axon guidance cues. Trends Neurosci 2002;25:173-175.

-2 Roncagliolo M, Benitez J, Eguibar JR: Progressive deterioration of central components of auditory brainstem responses during postnatal development of the myelin mutant taiep rat. Audiol Neurootol 2000;5:267-275.

-3 Kiernan BW, Garcion E, Ferguson J, Frost EE, Torres EM, Dunnett SB, Saga Y, Aizawa S, Faissner A, Kaur R, Franklin RJ, ffrench-Constant C: Myelination and behaviour of tenascin-C null transgenic mice. Eur $\mathrm{J}$ Neurosci 1999;11:3082-3092.

-4 Cork LC, Clarkson TB, Jacoby RO, Gaertner DJ, Leary SL, Linn JM, Pakes SP, Ringler DH, Stranderg JD, Swindle MM: The costs of animal research: Origins and options. Science 1997;276:758-759.

$\checkmark 5$ Swindle MM, Smith AC, Goodrich JA: Chronic cannulation and fistulization procedures in swine: A review and recommendations. J Invest Surg 1998;11:7-20.

-6 Barkovich AJ, Kjos BO, Jackson DE, Norman $D$ : Normal maturation of the neonatal and infant brain: MR imaging at $1.5 \mathrm{~T}$. Radiology 1988; 166:173-180.

7 van der Knaap MS, Valk J: MR imaging of the various stages of normal myelination during the first year of life. Neuroradiology 1990;31: 459-470.

-8 Koenig SH, Brown RD 3rd, Spiller M, Lundbom N: Relaxometry of brain: Why white matter appears bright in MRI. Magn Reson Med 1990;14:482-495.

-9 Martin E, Krassnitzer S, Kaelin P, Boesch C: MR imaging of the brainstem: Normal postnatal development. Neuroradiology 1991;33: 391-395.
10 Staudt M, Schropp C, Staudt F, Obletter N, Bise K, Breit A: Myelination of the brain in MRI: A staging system. Pediatr Radiol 1993; 23:169-176.

11 Huppi PS, Maier SE, Peled S, Zientara GP, Barnes PD, Jolesz FA, Volpe JJ: Microstructural development of human newborn cerebral white matter assessed in vivo by diffusion tensor magnetic resonance imaging. Pediatr Res 1998;44:584-590.

12 Nakagawa H, Iwasaki S, Kichikawa K, Fukusumi A, Taoka T, Ohishi H, Uchida H: Normal myelination of anatomic nerve fiber bundles: MR analysis. Am J Neuroradiol 1998;19: 1129-1136.

13 Sampaio R, Truwit CL: Myelination in the developing human brain; in Nelson CA, Luciana M (eds): Handbook of Developmental Cognitive Neuroscience. Cambridge, Bradford Books, 2001, pp 35-44.

14 Prayer D, Roberts T, Barkovich AJ, Prayer L, Kucharczyk J, Moseley M, Arieff A: Diffusionweighted MRI of myelination in the rat brain following treatment with gonadal hormones. Neuroradiology 1997;39:320-325.

$\checkmark 15$ Paus T, Collins DL, Evans AC, Leonard G, Pike B, Zijdenbos A: Maturation of white matter in the human brain: A review of magnetic resonance studies. Brain Res Bull 2001;54: 255-266.

16 Ashikaga R, Araki Y, Ono Y, Nishimura Y, Ishida O: Appearance of normal brain maturation on fluid-attenuated inversion-recovery (FLAIR) MR images. Am J Neuroradiol 1999; 20:427-431.

17 Baratti C, Barnett AS, Pierpaoli C: Comparative MR imaging study of brain maturation in kittens with $\mathrm{T} 1, \mathrm{~T} 2$, and the trace of the diffusion tensor. Radiology 1999;210:133-142.
18 Sie LT, van der Knapp MS, van Wezel-Meijler $\mathrm{G}$, Valk J: MRI assessment of myelination of motor and sensory pathways in the brain of preterm and term-born infants. Neuropediatrics 1997;28:97-105

19 Klingberg T, Vaidya CJ, Gabrieli JD, Moseley ME, Hedehus M: Myelination and organization of the frontal white matter in children: A diffusion tensor MRI study. Neuroreport 1999; 10:2817-2821.

20 van Wezel-Meijler G, van der Knapp MS: Diagnostic imaging of brain maturation in premature infants (in Dutch). Ned Tijdschr Geneeskd 2001;145:410-417.

21 Huisman TA, Wisser J, Martin E, Kubik-Huch R, Marincek B: Fetal magnetic resonance imaging of the central nervous system: A pictorial essay. Eur Radiol 2002;12:1952-1961.

22 Morgan B, Finan A, Yarnold R, Petersen S, Horsfield M, Rickett A, Wailoo M: Assessment of infant physiology and neuronal development using magnetic resonance imaging. Child Care Health Dev 2002;28(suppl 1):7-10.

$\checkmark 23$ Garel C, Chantrel E, Elmaleh M, Brisse H, Sebag G: Fetal MRI: Normal gestational landmarks for cerebral biometry, gyration and myelination. Childs Nerv Syst 2003;19:422-425.

24 Korogi Y, Takahashi M, Sumi M, Hirai T, Sakamoto Y, Ikushima I, Miyayama H: MR signal intensity of the perirolandic cortex in the neonate and infant. Neuroradiology 1996;38: 578-584.

25 Kinney H, Brody B, Kloman A, Gilles F: Sequence of central nervous system myelination in brain infancy. Patterns of myelination in autopsied infants. J Neuropathol Exp Neurol 1988;47:217-234. 